\title{
Immunohistochemical Expression of Surviving in Viral Hepatitis and Hepatocellular Carcinoma, a Comparative Study
}

\author{
Emadeldin R. Matar ${ }^{1}$, Mohammed H. Goda ${ }^{2}$ \\ ${ }^{1}$ Assistant Professor of Pathology, Faculty of Medicine, Al Azhar University, Cairo, Egypt \\ ${ }^{2}$ Lecturer of Surgery, Faculty of Medicine, Ain Shams University, Cairo, Egypt \\ Email: *Emad_rshde61@hotmail.com
}

How to cite this paper: Matar, E.R. and Goda, M.H. (2021) Immunohistochemical Expression of Surviving in Viral Hepatitis and Hepatocellular Carcinoma, a Comparative Study. Open Journal of Pathology, 11, 85-99.

https://doi.org/10.4236/ojpathology.2021.1 $\underline{14008}$

Received: April 18, 2021

Accepted: July 25, 2021

Published: July 28, 2021

Copyright $\odot 2021$ by author(s) and Scientific Research Publishing Inc. This work is licensed under the Creative Commons Attribution International License (CC BY 4.0).

http://creativecommons.org/licenses/by/4.0/

\begin{abstract}
Hepatocellular carcinoma (HCC) is the fifth most common neoplasm, the major cause of death in patients with cirrhosis, and the third most common cause of cancer-related death in the world. In Egypt, hepatocellular carcinoma (HCC) is the second most common cancer in men and the 6th most common cancers in women. Egypt has the highest prevalence of $\mathrm{HCV}$ in the world and the prevalence of HCC is increasing in the last years. Nowadays, HCV infection and its complications are among the leading public health challenges in Egypt with $13.8 \%$ of population infected and, in these patients, the risk of HCC is increased 17-fold. Glypican-3 (GPC3) is a member of heparin sulfate proteoglycan family which is linked to cell surface. It plays an important role in regulation of cell growth, differentiation, and migration. It is normally expressed in fetal liver and placenta but not in normal adult liver tissue. 8-Hydroxydeoxyguanosine (8-OHdG) is an oxidatively modified promutagenic DNA that is produced by oxygen radicals and is recognized as a useful marker in estimating DNA damage induced by oxidative stress. The present work is a retrospective, cross-sectional, study performed on 150 liver paraffin blocks that included 100 cases of chronic hepatitis $\mathrm{C}$ and 50 cases of hepatocellular carcinoma. These cases were collected from the Pathology Department. The 100 cases of chronic hepatitis $\mathrm{C}$ were graded into low grade chronic hepatitis (58) cases and high-grade chronic hepatitis (42) cases, 30 patients had cirrhosis, all in the high-grade group. The 50 cases of hepatocellular carcinoma (HCC) included 18 cases of well-differentiated HCC (grade I), 22 cases of moderately differentiated HCC (grade II) and 10 cases of poorly differentiated HCC (grade III). Among 50 cases of HCC, 44 cases showed cirrhosis in the non-neoplastic liver tissue. There was a statistically significant difference in $8-\mathrm{OHdG}$ index between chronic hepatitis and hepatocellular
\end{abstract}


carcinoma $(\mathrm{P}=0.006)$.

\section{Keywords}

Immunohistochemical, Viral Hepatitis, Hepatocellular Carcinoma

\section{Introduction}

Hepatocellular carcinoma (HCC) accounts for $>5 \%$ of all human cancers and for $80 \%-90 \%$ of primary liver cancers. It is a major health problem worldwide being the fifth most common malignancy in men, the eighth in women and the third most common cause of cancer-related death in the world [1].

HCC incidence has increased sharply over recent decades and has been attributed to chronic hepatitis $\mathrm{C}$ virus (HCV) infection. Chronic HCV infection, therefore, is a major risk factor for HCC development. Indeed, each year, 4-5\% of patients with chronic hepatitis C develop HCC. HCV infection increases the risk for HCC development by an estimated 17-fold compared to healthy individual [2].

In Egypt, the rates of $\mathrm{HCV}$ infection are among the highest in the world with a prevalence rate of up to 20\% [3] [4] [5], and recent investigations in Egypt have shown the increasing importance of HCV infection in the etiology of liver cancer, estimated to account for $40 \%-50 \%$ of cases [6].

The National Cancer Institute Pathology Registry in Egypt indicated that liver cancer formed $11.75 \%$ of the malignancies of all digestive organs and $1.68 \%$ of total malignancies and that liver tumors, were mostly HCC (70.48\%) [7].

Glypican-3 (GPC3) is a member of heparin sulfate proteoglycan family which is linked to cell surface. It plays an important role in regulation of cell growth, differentiation, and migration and is normally expressed in fetal liver and placenta but not in normal adult liver tissue [7].

The gene encoding Glypican-3 (GPC3) located on Xq26 and regulates several signaling pathways involving fibroblast growth factors and bone morphogenetic proteins [8].

Data have demonstrated a high incidence of GPC3 expression in HCC and suggested its implications in detecting malignant hepatic lesions and it is widely recognized as an efficient serological and histochemical marker for early hepatocellular carcinoma [9].

8-Hydroxydeoxyguanosine (8-OHdG) is an oxidatively modified promutagenic DNA that is produced by oxygen radicals and is recognized as a useful marker in estimating DNA damage induced by oxidative stress and in patients with chronic hepatitis $\mathrm{C}$, increased $8-\mathrm{OHdG}$ in DNA extracted from liver tissue was also reported [10].

It was found that oxidative DNA damage is associated with increased risk for HCC and hepatic 8-OHdG levels are useful as markers to identify the extreme 
high-risk subgroup [11].

\section{Aim of the Work}

The aim of this work is to evaluate glypican-3 (GPC-3) and

8-hydroxydeoxyguanosine (8-OhdG) expression in cases of chronic hepatitis $\mathrm{C}$ and hepatocellular carcinoma as predictive markers for hepatocellular carcinoma.

\section{Materials and Methods}

\subsection{Materials}

This work is a retrospective, cross-sectional study carried out on 150 liver paraffin blocks that were previously diagnosed as chronic hepatitis C (100 cases) and hepatocellular carcinoma (50 cases), collected from the Pathology Department, Faculty of Medicine, in the period from October 2019 to January 2020. The selected specimens were obtained by needle biopsy and surgical excision (Figures 1-5).

\subsection{Methods}

\section{I-Clinical evaluation:}

The clinical data concerning age and sex were obtained from the patient's files.

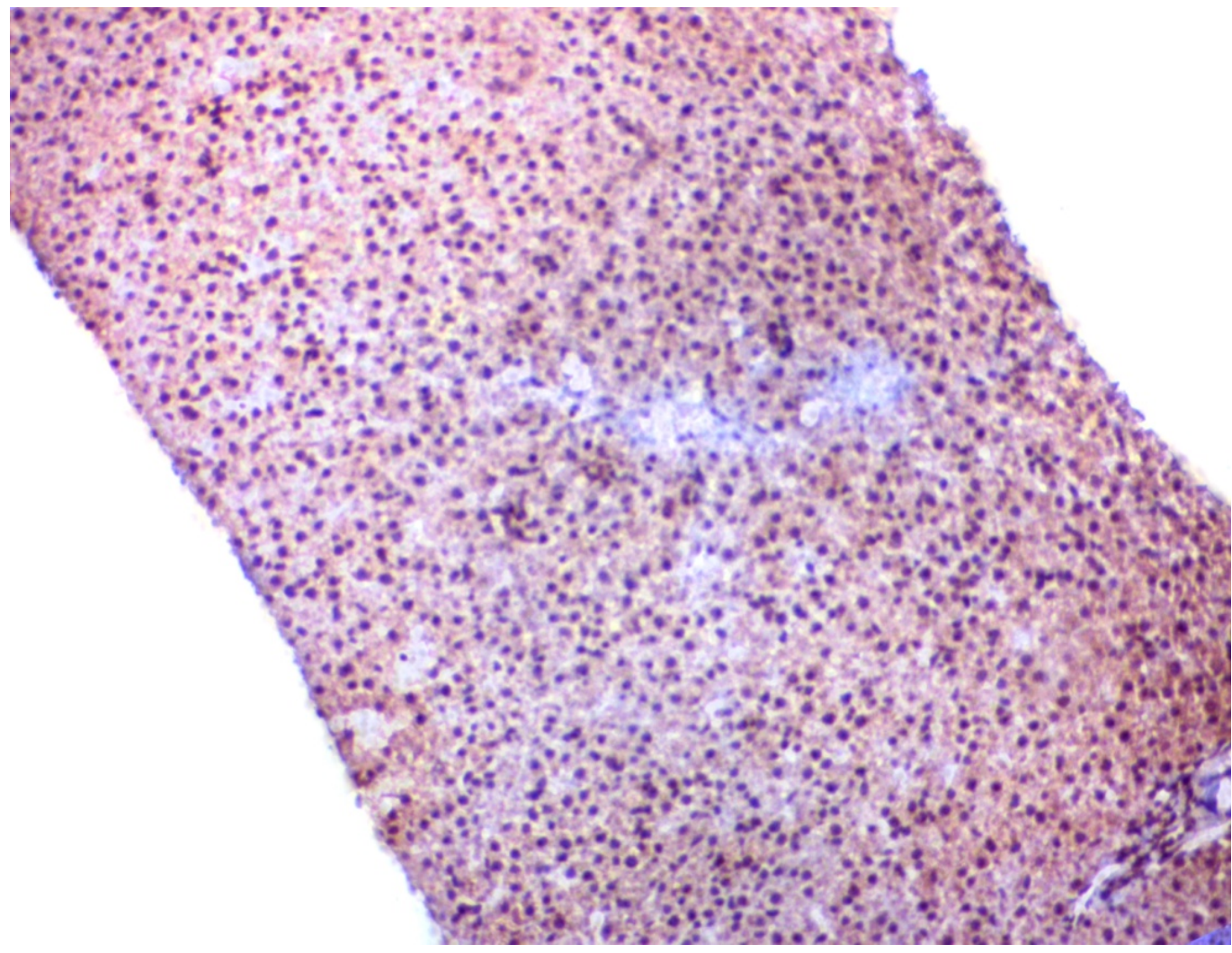

Figure 1. A core needle biopsy of chronic hepatitis C (A1, F1) showing low index 8-nuclear OHdG immunoreactivity. 


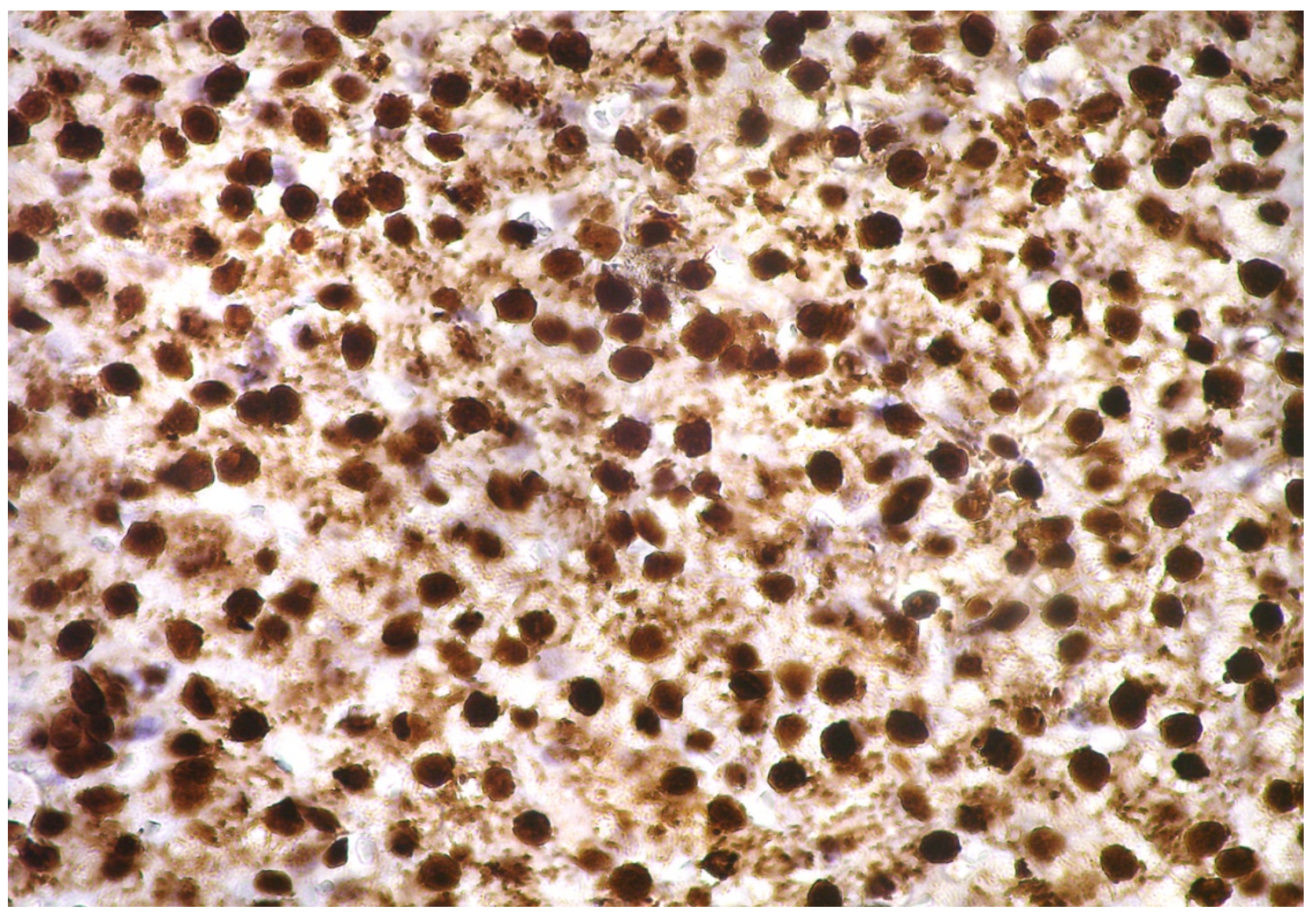

Figure 2. A core needle biopsy of chronic hepatitis C (A1, F1) showing high index 8-OHdG nuclear immunoreactivity. (immunoperoxidase stain, $\times 400$ ).

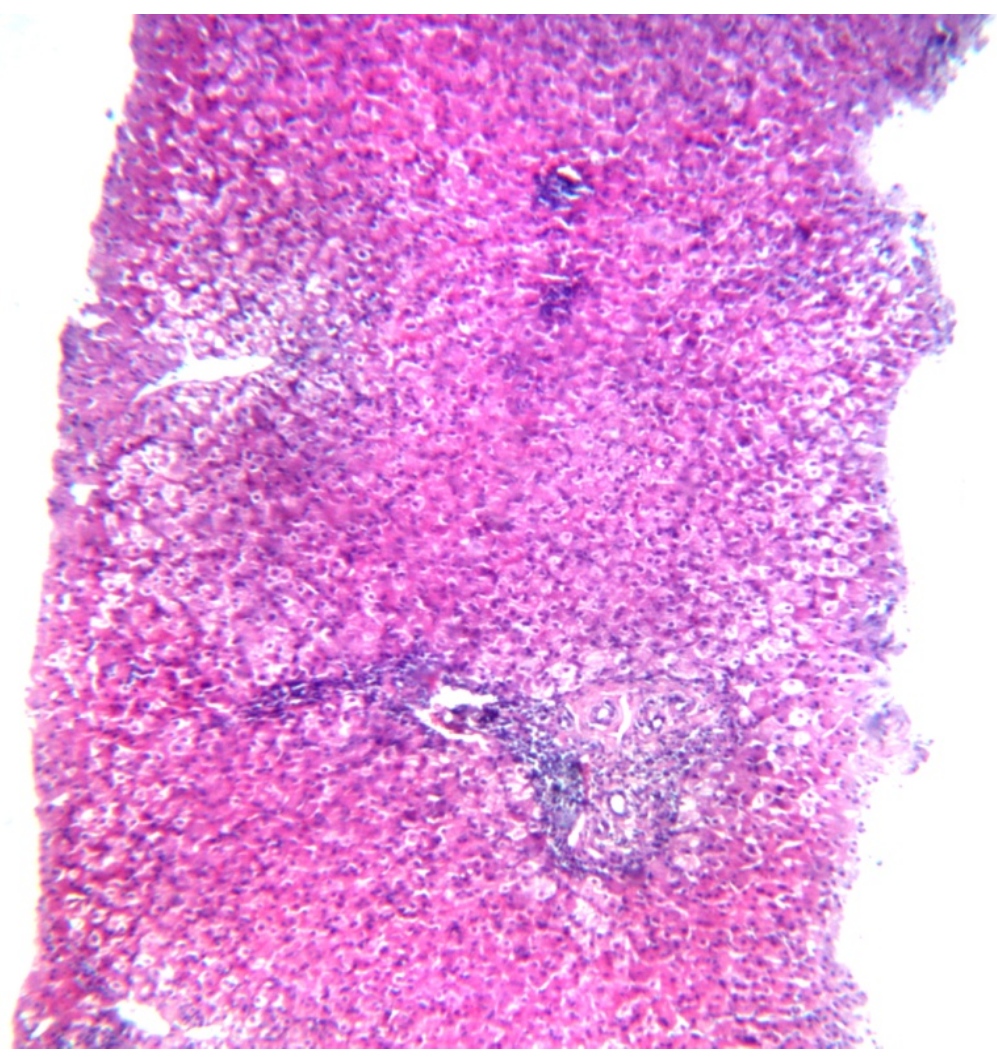

Figure 3. A core needle biopsy of chronic hepatitis C (A2, F2) (Hx \& E, ×100). 


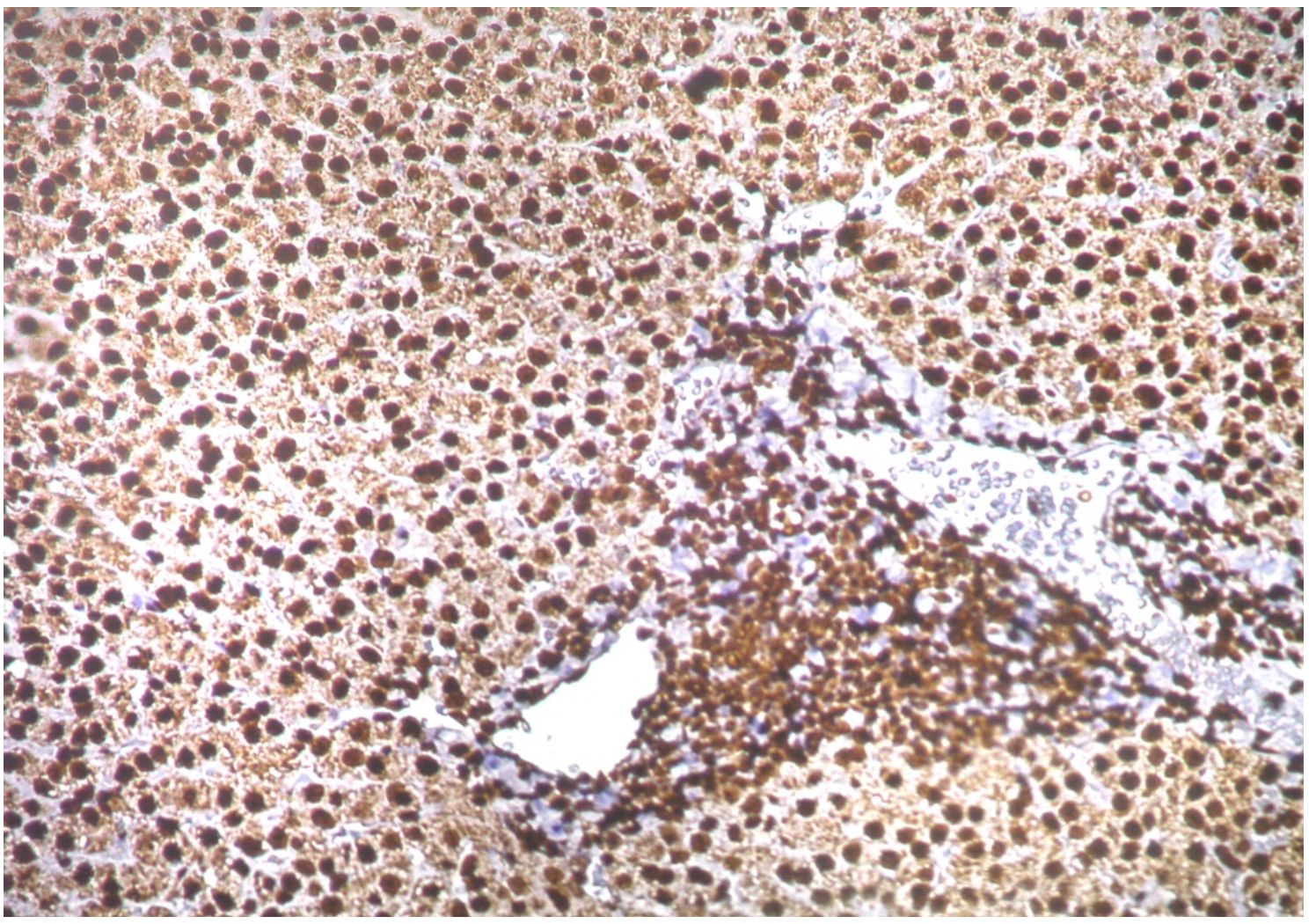

Figure 4. A core needle biopsy of chronic hepatitis C (A2, F2) showing high index 8-OHdG nuclear immunoreactivity, also immunoreactivity of chronic inflammatory cell (immunoperoxidase stain, $\times 200$ ).

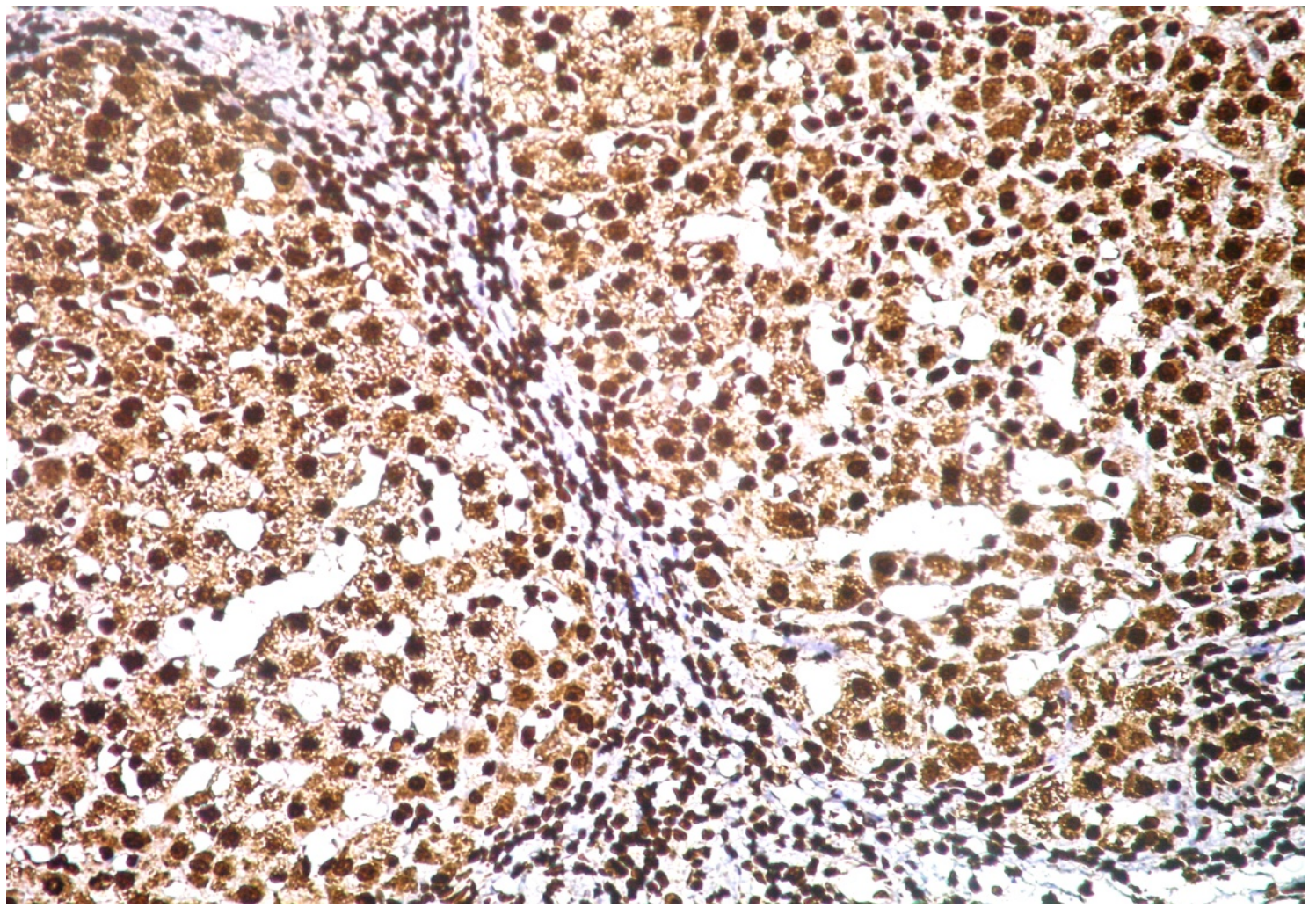

Figure 5. Higher magnification of previous image (Immunoperoxidase stain, $\times 300$ ). 


\section{II-Histopathological evaluation:}

Paraffin blocks of all cases were sectioned at 3 - 4-micron thickness and stained with routine hematoxylin and eosin stain to re-evaluate and confirm the diagnosis.

A) The histological criteria of HCV were assessed according to:

1-Ishak system

2-METAVIR system

HCC were graded into well, moderately and poorly differentiated according to WHO.

\section{III-Immunohistochemical evaluation: I-Primary antibodies:}

- Glypican-3(GPC-3): Mouse monoclonal antibody, Dilution 1:100; (CM396, A, B, Clone IG12, Biocare Medical LLC, Concord, USA).

- 8-Hydroxydeoxyguanosine(8-OHdG): Monoclonal antibody, Dilution1:100 (Clone15A3, SC-66036, Santa Cruz, California, USA).

\section{II-Universal kit:}

Super sensitive ${ }^{\mathrm{mx}}$ link-table IHC detection system. An improved Biotin-Streptvidin amplified(B-SA) detection system.

(Code No. QD000-5L Multilink ${ }^{\circledR}$. Detection kit, HRP, DAB, BioGenex, CA, U.S.A) designated to detect specific antigens in formalin-fixed, paraffin-embedded tissue using a modified labeled avidin-biotin technique.

The universal kit includes the following materials:

1) Peroxide block: one vial $(6 \mathrm{ml})$ of $3 \%$ hydrogen peroxide in water.

2) Link: one vial $(6 \mathrm{ml})$ of prediluted biotinylated, anti-immunoglobulins for mouse, rabbit, guinea pig and rat in phosphate buffered saline (PBS) with carrier protein and less than $0.09 \%$ sodium azide.

3) Label: one vial $(6 \mathrm{ml})$ of ready-to-use horse radish Peroxidase-conjugated (HRP) streptavidin in phosphate buffer saline (PBS) with carrier protein and less than $0.1 \%$ Procilin 300 .

4) Chromogen: one vial (2 ml) of 3,3-diamminobenzidin (DAB) chromogen solution.

5) Substrate: six vials ( $2.5 \mathrm{ml}$ each) of ready-to-use substrate buffer and one vial $(2 \mathrm{ml})$ of hydrogen peroxide Substrate solution.

2-Immunohistochemical procedure according to (Bancroft and Gamble, 2002):

1) Paraffin sections were cut at $5 \mu$ thickness and mounted on positively charged glass slides coated with poly L-lysine.

2) Paraffin sections were deparaffinized by incubating them in the oven at 56 C for 15 minutes then inserted in xylene for 30 minutes.

3) Rehydration of the slides in descending grades of alcohol: alcohol $95 \%$ for 5 minutes, alcohol $85 \%$ for 5 minutes and alcohol $75 \%$ for 5 minutes.

4) Slides were rinsed in distilled water for 5 minutes.

5) Washing the sections by PBS for 5 minutes. 
6) Antigen Retrieval: sections were immersed in ready to use Dako target retrieval solution ( $\mathrm{PH} \mathrm{6.0)}$, then boiled in this solution in a microwave for $20 \mathrm{mi}$ nutes.

7) Tapping off excess liquid using lintless tissue (such as gauze pad), carefully wipe around the specimen to remove any remaining liquid and to keep reagents within the prescribed area.

8) Application of 3\% Hydrogen peroxide to cover tissue sections for blocking endogenous peroxidase, incubated 5 minutes.

9) Rinsed gently with distilled water.

10) Tapping off excess liquid and wipping the slides as before.

11) 2 - 3 drops of primary antibody (Glypican-3 and 8-hydroxydeoxyguanosine) were placed on each slide and completely cover the specimen.

12) The slides were incubated in humidity chamber overnight at $2-8 \mathrm{C}$.

13) The slides were gently rinsed with buffer solution with avoiding flow directly on tissue.

14) Tapping off excess buffer immediately and wipe slides as before.

15) Sections were then incubated with biotinylated antimouse immunoglobulins for 15 minutes at room temperature followed by washing in buffer.

16) Sections were incubated with streptavidin-HRP for 15 minutes followed by washing.

17) Diamminobenzidin substrate was added to tissue sections; incubated for 5-10 minutes, then rinsed gently with distilled water.

18) Slides were immersed in a bath of Mayer's hematoxylin (M.H); incubated for $(2$ - 5) minutes, depending on the strength of the used hematoxylin.

19) Slides were gently rinsed in a distilled water bath.

20) Slides were dipped 10 times into a bath of ammonia water to remove excess used hematoxylin stain.

21) Slides were rinsed in a bath of distilled water for (2 - 5) minutes.

22) Slides were cleared in xylene for 3 changes and finely mount with a cover slip using DPX.

23) Positive and negative controls were stained at the same staining setting with the studied cases: Chronic hepatitis $c$ and hepatocellular carcinoma were used as positive control for $8-\mathrm{OHdG}$ and glypican3 respectively, while negative controls were done using the same tissue omitting the primary antibody.

Evaluation of the results of immunohistochemical staining

\section{1-Glypican-3 immunostaining \\ 2-8-Hydroxydeoxyguanosine}

The slides were evaluated by three different pathologists working separately. All discrepancies were discussed, and consensus reached.

\section{Statistical Analysis}

Data were coded and entered to EPI-INFO file using EPI-INFO version 6.1 computer package. 


\section{Results}

This work is a retrospective, cross-sectional study carried out on 150 liver paraffin blocks that were previously diagnosed as chronic hepatitis $\mathrm{C}$ (100 cases) and hepatocellular carcinoma (50 cases),

\section{I-Histopathological results of the studied cases:}

Histopathological re-evaluation was performed to confirm the diagnosis.

In 6 cases of HCC, cirrhosis couldn't be detected (Table 1).

The majority of studied cases of HCC were moderately differentiated (grade II) (44\%) (Table 2) and cytoarchitecturally the majority were of the trabecular variant (56\%) (Table 3).

$30 \%$ of the studied cases of chronic hepatitis were cirrhotic (F4). $58 \%$ of the studied cases of chronic hepatitis were low grade while $42 \%$ were high grade (Table 4 ).

\section{II-Clinical results of the selected cases:}

The age of the studied cases of HCC ranged from 41 - 73 and the mean was 58. The majority of studied cases of HCC were between 50 - 59 (44\%).

The age of the studied cases of chronic hepatitis $C$ ranged from $28-62$ and the

Table 1. Histopathologic diagnosis of the studied cases (150 cases).

\begin{tabular}{ccc}
\hline Histological diagnosis & No & $\%$ \\
\hline Hepatocellular carcinoma (HCC): & 50 & 33.3 \\
With cirrhosis & 44 & 12 \\
Without cirrhosis & 68 \\
Chronic hepatitis: & 100 & 66.7 \\
With cirrhosis & 30 & 30 \\
Without cirrhosis & 70 & 70 \\
Total & 150 & $100 \%$ \\
\hline
\end{tabular}

Table 2. Grading of Hepatocellular carcinoma.

\begin{tabular}{ccc}
\hline & No & $\%$ \\
\hline Well differentiated HCC (Grade I) & 18 & 36 \\
Moderately differentiated HCC (Grade II) & 22 & 44 \\
Poorly differentiated HCC (Grade III) & 10 & 20 \\
& $\mathbf{5 0}$ & $\mathbf{1 0 0 \%}$ \\
\hline
\end{tabular}

Table 3. Cytoarchitectural variant of Hepatocellular carcinoma.

\begin{tabular}{rcc}
\hline & No & $\%$ \\
\hline Trabecular HCC & 28 & 56 \\
Acinar HCC & 12 & 24 \\
Solid HCC & 8 & 16 \\
Clear HCC & 2 & 4 \\
& $\mathbf{5 0}$ & $\mathbf{1 0 0 \%}$ \\
\hline
\end{tabular}


Table 4. Staging and grading of chronic hepatitis according to Metavir.

\begin{tabular}{|c|c|c|c|c|c|c|c|c|c|c|c|}
\hline & & & & & & & & & Stage & & \\
\hline & & 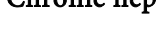 & & & & & F1 & F2 & F3 & F4 & Total \\
\hline & & No & & & & & 16 & 22 & 32 & 30 & 100 \\
\hline & & $\%$ & & & & & 16.0 & 22.0 & 32.0 & 30.0 & 100.0 \\
\hline & Low gr & nic hepatitis & & gh gr & ade $c$ & hronic & hepat & itis & & & \\
\hline $\begin{array}{l}\text { Chronic } \\
\text { hepatitis }\end{array}$ & & & & 2 & & A3 & To & & & Total & \\
\hline & No & $\%$ & No & $\%$ & No & $\%$ & No & $\%$ & No & & $\%$ \\
\hline & 38 & 58.0 & 18 & 18.0 & 24 & 24.0 & 42 & 42.0 & 100 & & 100 \\
\hline
\end{tabular}

Table 5. Age and sex distribution among the studied cases of chronic hepatitis.

\begin{tabular}{|c|c|c|c|c|c|c|c|c|c|c|c|}
\hline \multirow[b]{3}{*}{ Age } & \multicolumn{9}{|c|}{ Chronic hepatitis } & \multirow{2}{*}{\multicolumn{2}{|c|}{$\mathrm{HCC}$}} \\
\hline & \multicolumn{3}{|c|}{ Without cirrhosis } & \multicolumn{2}{|c|}{ With cirrhosis } & \multicolumn{4}{|c|}{ Total } & & \\
\hline & No & $\%$ & & No & $\%$ & No & & $\%$ & & NO & $\%$ \\
\hline $0-39$ & 40 & 57.15 & & 8 & 26.7 & 48 & & 48.0 & & 0 & 0 \\
\hline $40-49$ & 26 & 37.15 & & 16 & 53.3 & 42 & & 42.0 & & 10 & 20 \\
\hline $50-59$ & 4 & 5.7 & & 4 & 13.3 & 8 & & 8.0 & & 11 & 44 \\
\hline$\geq 60$ & 0 & 0.0 & & 2 & 6.7 & 2 & & 2.0 & & 18 & 36 \\
\hline \multirow[t]{4}{*}{ total } & 70 & 100 & & 30 & 100 & 100 & & 100 & & 50 & 100 \\
\hline & \multicolumn{6}{|c|}{ Chronic hepatitis } & & & & \multirow{3}{*}{$\mathrm{X}^{2}$} & \multirow{3}{*}{$\mathbf{P}$} \\
\hline & \multicolumn{2}{|c|}{ Without cirrhosis } & \multicolumn{2}{|c|}{ With cirrhosis } & \multicolumn{2}{|c|}{ Total } & Iepat & twais & achoma & & \\
\hline & No & $\%$ & No & $\%$ & No & $\%$ & No & & $\%$ & & \\
\hline Male & 46 & 65.7 & 20 & 66.7 & 102 & 68.0 & 36 & & 72.0 & \multirow{3}{*}{0.28} & \\
\hline Female & 24 & 34.3 & 10 & 33.3 & 48 & 32.0 & 14 & & 28.0 & & \\
\hline Total & 70 & 46.7 & 30 & 20.0 & 150 & 100.0 & 50 & & 33.3 & & \\
\hline
\end{tabular}

mean was 40 year. The majority of studied cases of chronic hepatitis were below 39 years (48\%) (Table 5$)$.

High 8-OHdG index was observed in $82 \%$ of cases of chronic hepatitis and in $52 \%$ of cases of HCC while low index was observed in $18 \%$ of cases of chronic hepatitis and in $48 \%$ of cases of HCC (Table 6).

There was a statistically significant difference in 8-OHdG index between chronic hepatitis and hepatocellular carcinoma $(\mathrm{P}=0.006)$ (Table 7).

\section{Discussion}

Hepatocellular carcinoma (HCC) is the fifth most common neoplasm, the major cause of death in patients with cirrhosis, and the third most common cause of cancer-related death in the world. In most countries, HCC accounts for 70\% $85 \%$ of primary liver cancer cases, with the burden of disease expected to increase in coming years [12]. 
Table 6. Summary of Immunohistochemical reactivity pattern of GPC-3 expression in hepatocellular lesions.

\begin{tabular}{|c|c|c|c|c|c|c|c|c|c|c|c|c|c|}
\hline & \multirow{3}{*}{ No } & \multicolumn{12}{|c|}{ GPC-3 expression } \\
\hline & & \multirow[b]{2}{*}{$\mathbf{N}$} & 0 & \multicolumn{2}{|c|}{+} & \multicolumn{2}{|c|}{++} & \multicolumn{2}{|c|}{+++} & \multicolumn{2}{|c|}{ Total (-) } & \multicolumn{2}{|c|}{ Total(+) } \\
\hline & & & $\%$ & No & $\%$ & No & $\%$ & No & $\%$ & No & $\%$ & & \\
\hline Low grade hepatitis & 58 & 58 & $100.0^{*}$ & 0 & 0.0 & 0 & 0.0 & 0 & 0.0 & 58 & $100.0^{*}$ & 0 & 0.0 \\
\hline High grade hepatitis & 42 & 24 & 57.1 & 10 & 23.8 & 4 & 9.5 & 4 & 9.5 & 24 & 57.1 & 18 & 42.9 \\
\hline Cirrhosis with carcinoma & 44 & 28 & 63.6 & 8 & 18.2 & 6 & 13.6 & 2 & 4.5 & 28 & 63.6 & 16 & 36.4 \\
\hline Cirrhosis without carcinoma & 30 & 20 & 60.0 & 4 & 13.3 & 4 & 13.3 & 2 & 6.7 & 20 & 20.0 & 10 & 33.3 \\
\hline Hepatocellular carcinoma & 50 & 6 & 12.0 & 12 & 24.0 & $16^{*}$ & 32.0 & 16 & $32.0^{*}$ & 3 & 12.0 & 44 & 88.0 \\
\hline $\mathrm{X}^{2}$ & & & 44.05 & & 8.11 & & 12.2 & & 16.48 & & $44 .($ & & \\
\hline $\mathbf{P}$ & & & $<0.001$ & & 0.08 & & 0.015 & & 0.002 & & $<0.0$ & 001 & \\
\hline
\end{tabular}

Table 7. Summary of Immunohistochemical reactivity pattern of 8-OHdG expression in hepatocellular lesions.

\begin{tabular}{ccccccc}
\hline & & \multicolumn{3}{c}{ 8-OHdG expression } \\
\cline { 3 - 6 } & No & \multicolumn{2}{c}{ low index } & \multicolumn{2}{c}{ High index } \\
\cline { 3 - 7 } & & No & $\%$ & No & $\%$ \\
\hline Chronic hepatitis & 100 & 18 & 18.0 & 82 & 82.0 \\
Hepatocellular carcinoma & 50 & 24 & 48.0 & 26 & 52.0 \\
$\mathbf{X}^{2}$ & & & & 7.44 & \\
$\mathbf{P}$ & & & & & \\
& & & & 0.006 & \\
\hline
\end{tabular}

The present work was performed on 150 liver paraffin blocks that included 100 cases of chronic hepatitis $\mathrm{C}$ and 50 cases of hepatocellular carcinoma.

The 100 cases of chronic hepatitis $C$ were graded into low grade chronic hepatitis (58) cases and high-grade chronic hepatitis (42) cases, 30 patients had cirrhosis, all in the high-grade group. The 50 cases of hepatocellular carcinoma (HCC) included 18 cases well differentiated HCC (grade I), 22 cases moderately differentiated HCC (grade II) and 10 cases poorly differentiated HCC (grade III). Among 50 cases of HCC, 44 cases showed cirrhosis in the non-neoplastic liver tissue.

Glypican-3 (GPC-3) belongs to glypican family that is a group of heparan sulfate proteoglycans linked to the outer surface of cell membrane through a glycosyl-phosphatidy-linositol anchor. Depending on the cellular context, glypicans function can be stimulatory or inhibitory activity, or signaling. The expression of GPC3 is detected in placenta and fetal liver, but not in other normal organs. During hepatic carcinogenesis, GPC3 have been reported to re-appear in HCC and to be released into serum [8] [13].

In our study, glypican-3 was expressed in $18(18 \%)$ of 100 cases of chronic hepatitis, all of high-grade hepatitis (42.9\%) and none of low-grade chronic he- 
patitis. The staining pattern was exclusively cytoplasmic without staining of plasma membranes or canaliculi, as has been described in some HCCs. There was a statistically significant difference between GPC-3 expression in low- and high-grade hepatitis $(\mathrm{P}=0.0016)$. This result was consistent with Abdul-Al et al. (2008) who studied GPC-3 expression in 120 cases of chronic hepatitis C (60 low grade and 60 high grade). They found that $25(83.3 \%)$ of 60 cases of high-grade chronic hepatitis showed positive staining for GPC3. In contrast, all biopsies in the low-grade group were negative. The positive hepatocytes were present in small groups, usually haphazardly distributed but sometimes with periportal predominance [14].

The most likely explanation of this result is that this oncofetal protein can be produced by regenerating liver cells, may be in association with other factors. This may be supported by the significantly higher hepatocyte proliferation count among the high-grade inflammation group than the low-grade group.

In the present study, glypican-3 was expressed in $26(35.1 \%)$ of 74 cases of cirrhosis, 16/44 (36.4\%) of cirrhosis with carcinoma and 10/30 (33.3\%) of cirrhosis without carcinoma. Reactivation of GPC3 expression may represent an early molecular event in hepatocarcinogenesis, which precedes actual morphologic transformation. This result was different from Baumhoer et al. (2018) who found positivity in 11/95 (12\%) of liver cirrhosis [15].

In this work, there was no statistically significant difference in glypican-3 expression between cirrhosis with or without carcinoma $(\mathrm{P}=0.97)$. However, Wang et al. (2018) reported that among 67 HCCs with a cirrhotic background, focal GPC3 immunoreactivity was detected in a small proportion of the cirrhotic nodules in 11 (16.4\%) cases but not in cirrhotic livers without HCC [16].

Although the underlying mechanisms for selective GPC3 expression in cirrhotic nodules remains elusive, GPC3 expression in chronic hepatitis ranged from none to $83.3 \%$ [14].

Wang et al., 2018 explained that the GPC3-expressing hepatocytes in cirrhotic nodules may have already initiated the neoplastic process at the molecular level. It is possible; therefore, that reactivation of GPC3 expression may represent an early molecular event in hepatocarcinogenesis, which precedes actual morphologic transformation [16].

Gong et al. (2014) considered that the transition from premalignant lesions to small HCC is associated with a sharp increase in GPC3 expression in a majority of cases. Simultaneously, the occurrence of small focal positivity for GPC3 in liver cirrhosis indicated strongly HCC regardless of the percentage of positive cells for glypican-3 [17].

Prolonged active chronic inflammation and extensive fibrosis due to HCV are responsible for the development of multiple occurrences of HCC. High GPC3 expression rate has also been reported in HCV-related HCC patients [18].

Several studies have demonstrated that GPC3 is positive in most HCC, and positive immunostaining ranged from $52.5 \%$ to $100 \%$ of HCC (Yan et al., 2019). 
In the present study, glypican-3 was detected in 22 (88\%) of 25 cases of HCC including $77.7 \%$ (7/9) of well differentiated cases, $100 \%(11 / 11)$ of moderately differentiated cases, and $80 \%(4 / 5)$ of poorly differentiated cases. Reactivation of the fetal phenotype, which is common in malignant tumors, may explain the expression of GPC3 in malignant hepatocellular nodules [19].

GPC3 was reported to promote proliferation of HCC cells by stimulating Wnt pathway through facilitating the interaction between the Wnts and their signaling receptors the activation of this signaling pathway induces the cytosolic accumulation and nuclear translocation of the transcription factor B-catenin. In the nucleus B-catenin associates with members of transcription factors family and induces the expression of genes that stimulate cell cycle progression and cell survival. GPC-3 overexpression represents a mechanism by which Wnt activity can be stimulated in HCC and ectopic GPC-3 in HCC induces the expression of c-Myc, a well characterized target of caonical Wnt signaling. Consequently, canonical Wnt activity has been shown to play a role in the progression of HCC [20].

In our study, there was no statistically significant difference in glypican-3 expression between different grades of HCC (P value $=0.85)$. Also, Gong et al. (2014) reported that glypican-3 expression was not related to HCC differentiation [17].

Anatelli et al. (2018) explained this wide range of positivity by the fact that GPC3 expression can be highly heterogeneous, even within the same tumor. This has been evidenced by the focal staining observed in their study and some previous studies by others [21].

In general, GPC3 has been reported to interfere with different pathways and growth factors and has a tissue and stage-specific role in the development and tumor growth [8].

Ishiguro et al. (2018) showed that anti-GPC3 antibody can be used as a potential antitumor agent for human liver cancer and can provide a novel treatment option for liver-cancer patients with GPC3-positive tumors [18].

ROS-induced DNA oxidation leads to a multitude of modifications to DNA bases, with 8-hydroxydeoxyguanosine (8-OHdG) representing the most frequent one. 8-OHdG, a guanine adducts used as an index of DNA oxidative damage, induces a point mutation in the daughter DNA strands, accumulates in cell DNA and causes mispairing, thus demonstrating its mutagenic and potentially carcinogenic role [22].

In our study, 8-OHdG was observed in all cases of chronic hepatitis $\mathrm{C}$, with high labeling index in $82 / 100(82 \%)$ of cases and low index in 18/100 (18\%) of cases. The staining was seen mainly in nuclei of hepatocytes, bile duct cells, portal inflammatory cells, and occasionally in the nuclei of sinusoidal cells.

Similar results were reported by Mahmood et al. (2014) who studied the expression of 8-OHdG in eight patients with chronic hepatitis $\mathrm{C}$ and found 8-OHdG expression in the nuclei of hepatocytes in all cases [23].

In the present study, the nuclear expression of $8-\mathrm{OHdG}$ was found in bile duct 
cells in inflamed portal tracts. This is because chronic liver injury, especially chronic hepatitis $\mathrm{C}$, is sometimes accompanied by bile duct damage [24].

In the present study, liver 8-OHdG expression was correlated with hepatic inflammation and there was a statistically significant difference in 8-OHdG expression between low and high grade hepatitis $(\mathrm{P}=0.05)$. This was consistent with Fujita et al. (2018) who found that 8-OHdG expression was correlated with the grade of hepatitis $(\mathrm{P}=0.032)$.This correlation may indicate the direct involvement of hepatic oxidative stress in the pathogenesis and progression of liver cell injury in chronic viral hepatitis [10].

Our present results confirm the previous studies results of a possible link between oxidative DNA damage and active inflammation in chronic liver diseases. It is therefore possible to speculate that the continuous active inflammation which yields continuous DNA damage is one of the risk factors for hepatocarcinogenesis, and it is conceivable that the treatment of chronic liver diseases to suppress hepatic inflammation may have preventive benefits.

In fact, studies have suggested that continuous hepatitis with high transaminase activity after curable resection of HCC is an independent risk factor for new development of HCC, and that treatment of chronic hepatitis $\mathrm{C}$ with interferon alpha may diminish the incidence of HCC even when viremia is persistent [20].

Tanaka et al. (2018) studied hepatic 8-hydroxydeoxyguanosine (8-OHdG) in liver biopsy samples from 118 nai"ve patients of which, HCC was identified in 36 patients (30.5\%) after follow-up period (mean was $6.7 \pm 3.3$ years), hepatic 8-OHdG expression levels in patients who developed HCC were significantly higher than in those who did not develop HCC $(\mathrm{P}<0.0001)$ [25].

The presence of OS markers in HCC tissue as well as in non-HCC tissue indicates that OS is closely related to the development of HCC and that non-HCC tissue under OS may become carcinomatous in the future. So, early detection, continuous monitoring, and subsequent treatment of OS in HCV-related liver disease may prevent further liver damage and slow down or prevent HCC development [23].

\section{Conclusions}

1) Preventive measures:

a) HBV vaccine should be taken since it is highly immunogenic and effective.

b) Disposal syringes should be used only once then discarded to eliminate risk of $\mathrm{HCV}$ and $\mathrm{HBV}$ infection.

c) Avoid other routes of $\mathrm{HCV}$ and HBV infections e.g.: through screening of blood and avoid sharing personal items like razors and toothbrushes.

2) Under trials:

a) Efforts should be done to produce effective vaccines for HCV since it is a major worldwide and national health problem.

b) Surviving should be evaluated as a predictor of early malignant transforma- 
tion especially in presence of dysplastic and cirrhotic nodules.

c) Anti-survivin antibodies should be tried in HCC treatment and evaluated.

d) Expression of surviving in hepatitis should be extended at a larger scale and re-evaluated.

\section{Conflicts of Interest}

The authors declare no conflicts of interest regarding the publication of this paper.

\section{References}

[1] Castello, G., Costantini, S. and Scala, S. (2015) Targeting the Inflammation in HCVAssociated Hepatocellular Carcinoma: A Role in the Prevention and Treatment. Journal of Translational Medicine, 8, Article No. 109. https://doi.org/10.1186/1479-5876-8-109

[2] Bartosch, B. (2019) HCV and Liver Carbohydrate Metabolism. 7 th Annual Meeting the New Visby University Network on Hepatitis C, Tartu, 22 February 2010, 112-123.

[3] Arafa, N., El Hoseiny, M., Rekacewicz, C., et al. (2015) Changing Pattern of Hepatitis C Virus Spread in Rural Areas of Egypt. Journal of Hepatology, 43, 418-424. https://doi.org/10.1016/j.jhep.2005.03.021

[4] El-Gafaary, M.M., Rekacewicz, C., Abdel-Rahman, A.G., et al. (2005) Surveillance of Acute Hepatitis C in Cairo, Egypt. Journal of Medical Virology, 76, 520-525. https://doi.org/10.1002/jmv.20392

[5] Lehman, E., Soliman, A., Ismail, K., Hablas, A., Seifeldin, I., Ramadan, M., El-Hamzawy, H., Shoushtari, C. and Wilson, M. (2018) Patterns of Hepatocellular Carcinoma Incidence in Egypt from a Population-Based Cancer Registry. Hepatology Research, 38, 465-473. https://doi.org/10.1111/j.1872-034X.2007.00299.x

[6] Mokhtar, N., Iman, G. and Iman, A. (2017) Cancer Pathology Registry: 2003-2004, and Time Trend Analysis. National Cancer Institute, Cairo University, Cairo.

[7] Yan, B., Wei, J.J., Qian, Y.M., Zhao, X.L., Zhang, W.W., et al. (2011) Expression and Clinicopathologic Significance of Glypican 3 in Hepatocellular Carcinoma. Annals of Diagnostic Pathology, 15, 162-169. https://doi.org/10.1016/j.anndiagpath.2010.10.004

[8] Filmus, J. and Capurro, M. (2018) The Role of Glypican-3 in the Regulation of Body Size and Cancer. Cell Cycle, 7, 2787-2790. https://doi.org/10.4161/cc.7.18.6672

[9] Tatrai, P., Somoracz, A., Batmunkh, E., et al. (2019) Agrin and CD34 Immunohistochemistry for the Discrimination of Benign versus Malignant Hepatocellular Lesions. The American Journal of Surgical Pathology, 33, 874-885.

https://doi.org/10.1097/PAS.0b013e318194b3ea

[10] Fujita, N., Horiike, S., Sugimoto, R., Tanaka, H., Iwasa, M., Kobayashi, Y., Hasegawa, K., Ma, N., Kawanishi, S., Adachi, Y. and Kaito, M. (2017) Hepatic Oxidative DNA Damage Correlates with Iron Overload in Chronic Hepatitis C Patients. Free Radical Biology \& Medicine, 42, 353-362. https://doi.org/10.1016/j.freeradbiomed.2006.11.001

[11] Tanaka, H., Fujita, N., Sugimoto, R., Urawa, N., Horiike, S., Kobayashi, Y., Iwasa, M., Ma, N., Kawanishi, S., Watanabe, S., Kaito, M. and Takei, Y. (2018) Hepatic Oxidative DNA Damage Is Associated with Increased Risk for Hepatocellular Carcinoma in Chronic Hepatitis C. British Journal of Cancer, 98, 580-586. https://doi.org/10.1038/sj.bjc.6604204

[12] Shaker, M.K., Abdella, H.M., Khalifa, M.O. and El Dorr, A.K. (2013) Epidemiologi- 
cal Characteristics of Hepatocellular Carcinoma in Egypt: A Retrospective Analysis of 1313 Cases. Liver International, 33, 1601-1606. https://doi.org/10.1111/liv.12209

[13] Jia, H.L., Ye, Q.H., Qin, L.X., et al. (2017) Gene Expression Profiling Reveals Potential Biomarkers of Human Hepatocellular Carcinoma. Clinical Cancer Research, 13, 1133-1139. https://doi.org/10.1158/1078-0432.CCR-06-1025

[14] Abdul-Al, H.M., Makhlouf, H.R., Wang, G. and Goodman, Z.D. (2018) Glypican-3 Expression in Benign Liver Tissue with Active Hepatitis C: Implications for the Diagnosis of Hepatocellular Carcinoma. Human Pathology, 39, 209-212. https://doi.org/10.1016/j.humpath.2007.06.004

[15] Baumhoer, D., Tornillo, L., Stadlmann, S., Roncalli, M., Diamantis, E. and Terracciano, L. (2018) Glypican 3 Expression in Human Nonneoplastic, Preneoplastic, and Neoplastic Tissues. A Tissue Microarray Analysis of 4,387 Tissue Samples. American Journal of Clinical Pathology, 129, 899-906. https://doi.org/10.1309/HCQWPWD50XHD2DW6

[16] Wang, H., Anatelli, F., Zhai, Q., Adley, B., Chuang, S. and Yang, X. (2018) Glypican-3 as a Useful Diagnostic Marker That Distinguishes Hepatocellular Carcinoma from Benign Hepatocellular Mass Lesions. Archives of Pathology \& Laboratory Medicine, 132, 1723-1728. https://doi.org/10.5858/132.11.1723

[17] Gong, L., Wei, L., Ren, P., Zhang, W., Liu, X., Han, X., Yao, L., Zhu, S., Miao, L.M., Li, Y. and Zhang, W. (2014) Dysplastic Nodules with Glypican-3 Positive Immunostaining: A Risk for Early Hepatocellular Carcinoma. PLoS ONE, 9, e87120. https://doi.org/10.1371/journal.pone.0087120

[18] Yorita, K., Takahashi, N., Takai, H., Kato, A., Suzuki, M., Ishiguro, T., et al. (2019) Prognostic Significance of Circumferential Cell Surface Immunoreactivity of Glypican-3 in Hepatocellular Carcinoma. Liver International, 31, 120-131. https://doi.org/10.1111/j.1478-3231.2010.02359.x

[19] Yan, B.C., Gong, C., Song, J., et al. (2019) Arginase-1: A New Immunohistochemical Marker of Hepatocytes and Hepatocellular Neoplasms. The American Journal of Surgical Pathology, 34, 1147-1154. https://doi.org/10.1097/PAS.0b013e3181e5dffa

[20] Li, B., Liu, H., Shang, H., Li, P., Li, N. and Ding, H. (2012) Diagnostic Value of Glypican-3 in Alpha Fetoprotein Negative Hepatocellular Carcinoma Patients. African Health Sciences, 13, 703-709. https://doi.org/10.4314/ahs.v13i3.26

[21] Anatelli, F., Chuang, S.T., Yang, X.J. and Wang, H.L. (2008) Value of Glypican 3 Immunostaining in the Diagnosis of Hepatocellular Carcinoma on Needle Biopsy. American Journal of Clinical Pathology, 130, 219-223. https://doi.org/10.1309/WMB5PX57Y4P8QCTY

[22] Shigura, S.D. (2018) Hepatitis C Virus: Molecular Biology \& Current Therapeutic Options. Indian Journal of Medical Research, 131, 17-34.

[23] Cardin, R., Piciocchi, M., Bortolami, M., Kotsafti, A., Barzon, L., Lavezzo, E., et al. (2014) Oxidative Damage in the Progression of Chronic Liver Disease to Hepatocellular Carcinoma: An Intricate Pathway. World Journal of Gastroenterology, 20, 3078-3086. https://doi.org/10.3748/wjg.v20.i12.3078

[24] Mohamoud, Y., Mumtaz, G., Riome, S., Miller, D. and Abu-Raddad, L. (2013) The Epidemiology of Hepatitis C Virus in Egypt: A Systematic Review and Data Synthesis. BMC Infectious Diseases, 13, 288. https://doi.org/10.1186/1471-2334-13-288

[25] Kitada, T., Seki, S., Iwai, S., Yamada, T., Sakaguchi, H. and Wakasa, K. (2018) In Situ Detection of Oxidative DNA Damage, 8-Hydroxydeoxyguanosine, in Chronic Human Liver Disease. Journal of Hepatology, 35, 613-618. https://doi.org/10.1016/S0168-8278(01)00171-4 